\title{
FORCED HEAT CONVECTION IN LAMINAR FLOW THROUGH RECTANGULAR DUCTS*
}

\author{
BY \\ S. C. R. DENNIS, A. McD. MERCER ANd G. POOTS \\ The Queen's University of Belfast
}

Introduction. In this paper we consider the problem of finding the heat transfer to the wall of a duct which has a rectangular cross-section and through which a hot viscous fluid passes in steady established laminar motion. We shall make the usual simplifying assumptions that the thermal properties of the fluid are independent of temperature, that liquids are incompressible and that gases obey the perfect gas law. The first is strictly true only for small heat input and, of course, the assumption of established motion ignores the hydrodynamical boundary layer in the inlet. The problem is of engineering interest since, in many applications of gas-flow heat exchangers, flow passages are used which have small cross-section and a high ratio of surface area to core volume, so that the Reynolds number is often small enough for laminar flow to exist. Practical cross-sections can often be approximated by simple geometrical shapes and theoretical correlations of heat transfer with cross-section are of value in reducing the amount of practical test data required.

The rectangular cross-section gives rise to an essentially three-dimensional temperature distribution and has therefore received less attention than those involving twodimensional distributions, such as the circle and the case of infinite parallel walls. Some results have been given by Clark and Kays (1953) [1] by considering conditions far enough from the thermal inlet to assume a fully developed temperature profile, but this gives only asymptotic results and no information is obtained on the variation of heat transfer in the thermal entry region. On the other hand experimental data are given regarding this variation and it is therefore of interest to obtain theoretical results taking into account the undeveloped temperature profile. This is the object of this paper although it is hoped that the numerical analysis of the governing partial differential equation, which occurs in wider fields, will also be of interest.

Governing equations and basic thermal quantities. We consider a duct whose axis is the $\zeta$-axis of rectangular coordinates $(\xi, \eta, \zeta)$ and whose cross-section perimeter is, in general, the curve $C(\xi, \eta)=0$. The constant cross-sectional area is $A$ and the length of the perimeter is $S$. In customary notation the velocity field is $(u, v, w)$, but for steady established laminar motion under a constant pressure gradient $P / L$ we have $u=v=0$ and $w \equiv w(\xi, \eta)$ where

$$
\frac{\partial^{2} w}{\partial \xi^{2}}+\frac{\partial^{2} w}{\partial \eta^{2}}=-\frac{P}{\mu L},
$$

with $w=0$ on $C$. The energy equation governing the temperature $T(\xi, \eta, \zeta)$ of the fluid is, subject to the stated assumptions,

$$
\kappa\left(\frac{\partial^{2} T}{\partial \xi^{2}}+\frac{\partial^{2} T}{\partial \eta^{2}}+\frac{\partial^{2} T}{\partial \zeta}\right)=w \frac{\partial T}{\partial \zeta},
$$

${ }^{*}$ Received Nov. 5, 1957; revised manuscript received Sept. 26, 1958. 
where $\kappa$ is the thermometric conductivity, and second order terms, such as that due to internal heat generation, are neglected. The origin is at the thermal inlet and we suppose that the fluid enters $\zeta \geq 0$ with constant temperature $T_{0}$. We introduce dimensionless coordinates $x=\xi / d, y=\eta / d$ and $z=\zeta / d$ Pé where $d=4 A / S$ and Pé is the Péclet number, equal to the product of the Reynolds and Prandtl numbers, that is, $d w_{0} / \kappa$. The Reynolds number $d w_{0} \rho / \mu$ is based on the mean velocity $w_{0}$, that is, the ratio of total flow to cross-sectional area. It now appears that the ratio of axial conduction $\kappa \partial^{2} T / \partial \zeta^{2}$ to the axial convection term $w \partial T / \partial \zeta$ is of order $(1 / \mathrm{P} 6)^{2}$, so that axial conduction may be neglected for large enough Pé. This can lead to discrepancies in the special case of low Reynolds number flow of low Prandtl number fluids, such as certain liquid metals, but for water, air and high Prandtl number oils it is justified. Equations (1) and (2) then become

$$
\nabla_{1}^{2} w=-\frac{d^{2} P}{\mu L}
$$

and

$$
\nabla_{1}^{2} \theta=\frac{w}{w_{0}} \frac{\partial \theta}{\partial z}
$$

where

$$
\nabla_{1}^{2} \equiv \frac{\partial^{2}}{\partial x^{2}}+\frac{\partial^{2}}{\partial y^{2}} \quad \text { and } \quad \theta=\frac{T-T_{1}}{T_{0}-T_{1}},
$$

$T_{1}\left(<T_{0}\right)$ being a representative temperature associated with the duct wall in the region $\zeta>0$. The boundary condition for Eq. (3) is that $w=0$ on the transformed boundary $C^{\prime}(x, y)=0$ while that for Eq. (4) depends upon the assumptions that are made. If $T_{1}$ is taken as the wall temperature, assumed constant, we have $\theta=1$ within $C^{\prime}$ when $z=0$ and $\theta=0$ on $C^{\prime}$ when $z>0$. There is also another boundary condition in which we interpret $T_{1}$ as the temperature of the medium just outside the duct wall, again assumed constant. It has been shown by Hampton [2] that, when heat losses by radiation and natural convection take place from a body at temperature $T$ into surroundings at temperature $T_{1}$, the flux of heat $H\left(\mathrm{cal} / \mathrm{cm}^{2} / \mathrm{sec}\right)$ is well represented for temperatures from $0-400^{\circ} \mathrm{C}$. by the empirical formula

$$
H=A\left(T-T_{1}\right)+B\left(T-T_{1}\right)^{2} .
$$

The constants $A$ and $B$ depend upon the emissivity $E$ of the body, $A$ varying from $1.96 \times 10^{-4}$ when $E=1$ to $1.33 \times 10^{-4}$ when $E=0$ and $B$ varying correspondingly from $1.71 \times 10^{-6}$ to $0.25 \times 10^{-6}$. If we identify $T$ with the temperature of the duct wall (assumed ideally to be of negligible thickness so that $T$ is the temperature of the fluid in contact with it) the heat flux to the wall from the fluid is $H=-k \partial T / \partial \nu$, where $k$ is the thermal conductivity of the fluid and $\nu$ is the outward drawn normal from the duct wall. Substituting in Eq. (5) and introducing dimensionless quantities we obtain

$$
-\frac{k}{d} \frac{\partial \theta}{\partial \nu^{\prime}}=A \theta+B\left(T_{0}-T_{1}\right) \theta^{2},
$$

where $\nu^{\prime}=\nu / d$. Now $\theta<1$, tending to zero for large $z$, while $B$ is of order $10^{-2} A$ so that for small $T_{0}-T_{1}$ (which is implied in the basic assumptions) we may neglect the 
second term on the right hand side of Eq. (6). Putting $N=A d / k$, the complete boundary conditions for $\theta$ in this case may therefore be stated as

$$
\theta=1 \text { within } C^{\prime} \text { when } z=0, \partial \theta / \partial \nu^{\prime}=-N \theta \text { on } C^{\prime} \text { when } z>0 .
$$

If $N$ is infinite we get $T=T_{1}$ at the wall, so that this special case gives the constant wall temperature condition. If $N=0$ we have the trivial solution $T=T_{0}$. In practice $N$ must lie somewhere in between these limits.

The solution of Eq. (4) may be written

$$
\theta=\sum_{n=1}^{\infty} a_{n} \theta_{n}(x, y) \exp \left(-\lambda_{n} z\right), \quad(n=1,2,3 \cdots),
$$

where $\theta_{n}$ and $\lambda_{n}$ are eigenfunctions and eigenvalues of the membrane equation

$$
\nabla_{1}^{2} \theta+\frac{\lambda w(x, y)}{w_{0}} \Theta=0,
$$

subject to the boundary condition, deduced from the second of the conditions (7), that $\partial \theta / \partial v^{\prime}=-N \theta$ on $C^{\prime}$. The theory of this equation is well known and is dealt with, for example, by Courant and Hilbert [3]. Since $w(x, y)$ is positive within $C^{\prime}$ the eigenvalues $\lambda_{n}$ are real and positive and the eigenfunctions form a complete orthogonal set satisfying the property

$$
\iint_{D^{\prime}} w \Theta_{m} \Theta_{n} d x d y=0, \quad(m \neq n),
$$

where $D^{\prime}$ is the domain bounded by $C^{\prime}$. Each $\theta_{n}$ has arbitrary amplitude which we choose, for convenience, so that

$$
\iint_{D^{\prime}} w \Theta_{n}^{2} d x d y=\iint_{D^{\prime}} w d x d y
$$

Putting $z=0$ in Eq. (8) we have from first of the conditions (7) that

$$
1=\sum_{n=1}^{\infty} a_{n} \theta_{n}
$$

so that from Eqs. (10) and (11)

$$
\mathrm{Q}_{n}=\iint_{D^{\prime}} w \Theta_{n} d x d y / \iint_{D^{\prime}} w d x d y .
$$

The temperature $\theta(x, y, z)$ is therefore known to any desired accuracy once sufficient $\Theta_{n}$ have been found. Two further thermal quantities are of interest. Experimental measurements are made on the basis of a mean mixed temperature of the fluid, that is, $\theta(x, y, z)$ averaged with respect to the local fluid velocity over any section of the duct. This temperature is a function of $z$ only and its difference between any two sections gives a measure of the heat transferred across the wall between them. Denoting it by $T_{M}$ then $\theta_{M}=\left(T_{M}-T_{1}\right) /\left(T_{0}-T_{1}\right)$ and is given by

$$
\begin{aligned}
\theta_{M}(z) & =\iint_{D^{\prime}} w \theta d x d y / \iint_{D^{\prime}} w d x d y \\
& =\sum_{n=1}^{\infty} Q_{n}^{2} \exp \left(-\lambda_{n} z\right) .
\end{aligned}
$$


The remaining quantity to be considered is the rate of heat transfer per unit area to the wall of the duct, defined by means of a heat transfer coefficient. If $H$ is the heat flux to a given area of the duct wall, the fundamental equation defining such a coefficient, $h$, is $H=h \Delta T$, where $\Delta T$ is a representative temperature difference. We consider two coefficients, of somewhat different natures, obtained by different choices of $\Delta T$. First taking $\Delta T=T_{M}-T_{1}$ we obtain the local coefficient of heat transfer $h_{L}$, which measures the local average rate of heat transfer to the duct wall as a function of longitudinal distance down the duct. The total heat transferred to the area between the sections at $\zeta$ and $\zeta+d \zeta$ is $d q=h_{L} S d \zeta\left(T_{M}-T_{1}\right)=h_{L} S d \zeta \theta_{M}\left(T_{0}-T_{1}\right)$ and if $s$ is the distance measured along the perimeter of the boundary curve $C$ in an anti-clockwise direction we must also have

$$
d q=-k d \zeta \int_{c} \frac{\partial T}{\partial \nu} d s=-k\left(T_{0}-T_{1}\right) d \zeta \int_{C} \frac{\partial \theta}{\partial \nu} d s .
$$

We equate these two and introduce the appropriate dimensionless heat transfer coefficient or Nusselt number, defined as $h_{L} d / k$, so that we obtain for the local Nusselt number

$$
N u(z)=-d \int_{C^{\prime}} \frac{\partial \theta}{\partial \nu^{\prime}} d s^{\prime} / S \theta_{M},
$$

where $s^{\prime}=s / d$. We now eliminate $\theta$ in terms of the $\theta_{n}$ by Eq. (8) and apply Green's theorem to Eq. (9), so that

$$
\int_{C^{\prime}} \frac{\partial \Theta_{n}}{\partial \nu^{\prime}} d s^{\prime}=-\frac{\lambda_{n}}{w_{0}} \iint_{D^{\prime}} w \Theta_{n} d x d y
$$

Using Eq. (13) and since $\iint_{D^{\prime}} w d x d y=A^{\prime} w_{0}$, where $A^{\prime}$ is the dimensionless area within $C^{\prime}$, we finally obtain

$$
N u(z)=\frac{1}{4 \theta_{M}} \sum_{n=1}^{\infty} \lambda_{n} \Theta_{n} \exp \left(-\lambda_{n} z\right),
$$

where $\Theta_{n}=Q_{n}^{2}$. At large distances down the duct $N u(z)$ approaches a limiting minimum value. If $\lambda_{1}$ is the smallest of the $\lambda$ 's we have, as $z \rightarrow \infty$, that $4 \theta_{M} N u(z) \sim \lambda_{1} B_{1}$ exp $\left(-\lambda_{1} z\right)$ and $\theta_{M}(z) \sim \Theta_{1} \exp \left(-\lambda_{1} z\right)$ so that $N u(\infty)=\lambda_{1} / 4$. For experimental measurements a mean coefficient is generally more useful than the local coefficient. This is based on the total heat, $q$, transferred to the wall between the thermal inlet and the section $\zeta$. Definition of this coefficient again depends upon the choice of $\Delta T$ and the one most commonly used is the logarithmic mean temperature difference

$$
\Delta T_{l n}=\frac{\Delta T \max -\Delta T \min }{\ln (\Delta T \max )-\ln (\Delta T \min )}=\frac{\left(T_{0}-T_{1}\right)-\left(T_{M}-T_{1}\right)}{\ln \left(T_{0}-T_{1}\right)-\ln \left(T_{M}-T_{1}\right)} .
$$

Adopting this definition in the fundamental equation we have $q=h_{l_{n}} S \zeta \Delta T_{l_{n}}$. Now $q$ can either be obtained by integrating Eq. (15) from zero to $\zeta$ or, alternatively, it is the heat given up by the fluid in cooling from $T_{0}$ to $T_{M}$, so that $q=A w_{0} \rho C_{p}\left(T_{0}-T_{M}\right)$. Equating these two and introducing dimensionless quantities we have the mean logarithmic Nusselt number, $h_{l n} d / k$, given by

$$
N u^{\prime}(z)=\frac{1}{4 z} \ln \left(\frac{1}{\theta_{M}}\right) .
$$


The advantage of basing the mean Nusselt number on the logarithmic mean temperature difference is that $N u^{\prime}(z)$ tends to the same limiting value as the local coefficient $N u(z)$. For as $z \rightarrow \infty, \theta_{M} \sim \beta_{1} \exp \left(-\lambda_{1} z\right)$ and hence

$$
N u^{\prime}(z) \sim \frac{1}{4}\left\{\lambda_{1}+\frac{1}{z} \ln \left(\frac{1}{B_{1}}\right)\right\} .
$$

The foregoing results are based on fundamental definitions given by Jakob [4] and are true for a duct of any cross-section.

Basis of the method of solution. We consider the general domain $D^{\prime}$. The following is similar in principle to the method of Galerkin [5]. Let $\left\{\phi_{n}\right\}$ be the complete set of eigenfunctions of the equation

$$
\nabla_{1}^{2} \phi+\Lambda \phi=0
$$

with $\partial \phi / \partial \nu^{\prime}=-N \phi$ on $C^{\prime}$. Any arbitrary function $\Theta(x, y)$ which satisfies these boundary conditions and which possesses continuous partial derivatives up to the second order can be expanded in an absolutely and uniformly convergent series in the form

$$
\Theta(x, y)=\sum_{m=0}^{\infty} a_{m} \phi_{m}(x, y)
$$

where

$$
a_{m}=\frac{1}{\delta_{m}(m)} \iint_{D^{\prime}} \theta \phi_{m} d x d y
$$

and

$$
\delta_{p}(m)=\iint_{D^{\prime}} \phi_{m} \phi_{p} d x d y,
$$

so that $\delta_{p}(m)=0$ unless $m=p$. We can make $\theta$ the solution of Eq. (9) so that multiplying this equation by $\phi_{m}$ and integrating over $D^{\prime}$ we have, by Eqs. (21) and (23),

$$
\delta_{m}(m) \Lambda_{m} a_{m}=\lambda \iint_{D^{\prime}} r(x, y) \phi_{m} \theta d x d y, \quad(m=0,1,2, \cdots),
$$

where $r(x, y)=w(x, y) / w_{0}$. If we substitute for $\theta$ by Eq. (22) then Eq. (25) is reduced to an infinite set of homogeneous linear algebraic equations

$$
\sum_{p=0}^{\infty}\left\{\delta_{p}(m) \Lambda_{m}-\lambda b_{p}(m)\right\} a_{p}=0, \quad(m=0,1,2, \cdots),
$$

where

$$
b_{p}(m)=\iint_{D^{\prime}} r \phi_{m} \phi_{p} d x d y .
$$

The matrix associated with Eqs. (26) is symmetrical since $b_{p}(m) \equiv b_{m}(p)$ and the eliminant for a non-trivial solution gives an infinite determinantal equation $\Delta(\lambda)=0$ whose latent roots are the eigenvalues of Eq. (9). Dividing each row of $\Delta(\lambda)$ by its leading diagonal element, the resulting determinant converges [6] if the off-diagonal sum is absolutely convergent and $\lambda$ has no value which makes a leading diagonal element zero. If this condition is satisfied the convergence of $\sum_{p=0}^{\infty}\left|a_{p}\right|$ and, moreover, 
of $\sum_{p=0}^{\infty} \delta_{p}(p) \Lambda_{p}\left|a_{p}\right|$ follows. The eigenvectors $\left\{a_{p}^{(n)}\right\}$ corresponding to a given root $\lambda=\lambda_{n}$ can then be obtained, theoretically, in terms of any arbitrary coefficient but in practice the determination of a given eignensolution is a problem in numerical analysis. One special point concerning the above formulae may be noted. It will be necessary in the rectangular case to specify solutions of Eq. (21) by number pairs, that is $\left\{\phi_{m, n}\right\}$ rather than $\left\{\phi_{m}\right\}$, and the expansion (22) is now a sum over all number pairs from $m, n=0,1,2, \cdots$. Thus $b_{p}(m)$ in Eq. (26) is then written $b_{p, q}(m, n)$ and is associated with a coefficient $a_{p, q}$ in a double sum over number pairs from $p, q=0,1,2, \cdots$. The equations hold for $m, n=0,1,2, \cdots$, and $\delta_{p, q}(m, n)$, written for $\delta_{p}(m)$ in Eq. (24), is non-zero only if both $m=p$ and $n=q$.

The rectangular cross-section. In this case the boundary conditions become

$$
\frac{\partial \theta}{\partial x}= \pm N \Theta \quad \text { when } \quad x=\begin{aligned}
& 0 \\
& l
\end{aligned}, \quad \frac{\partial \theta}{\partial y}= \pm N \Theta \text { when } y=\begin{aligned}
& 0 \\
& l^{\prime}
\end{aligned},
$$

where $l=(1+\alpha) / 2, l^{\prime}=(1+1 / \alpha) / 2$ and $\alpha$ is the aspect ratio. Now the functions $X_{m}(x) \equiv \sin \left(\beta_{m} x+\beta_{m}^{\prime}\right)$, where $\tan \beta_{m}^{\prime}=\beta_{m} / N,\left(0 \leq \beta_{m}^{\prime} \leq \pi / 2\right)$, satisfy the first of Eqs. (28) if $\beta_{m}(m=0,1,2, \cdots)$, is a positive root (the negative roots only repeat the functions) of the equation

$$
\tan \beta l=2 N \beta /\left(\beta^{2}-N^{2}\right) .
$$

The roots of Eq. (29) form two separate sets which satisfy respectively the equations

and

$$
\beta \tan \frac{1}{2} \beta l=N
$$

$$
N \tan \frac{1}{2} \beta l+\beta=0,
$$

the corresponding functions being respectively symmetrical and anti-symmetrical about $x=l / 2$. The $\operatorname{root} \beta=0$ of the second equation does not contribute a function $X_{m}(x)$ but the root $\beta=0$ of the first in the case $N=0$ contributes a function $X_{m}(x)=1$, which must be included for completeness. A similar set of functions $Y_{n}(y) \equiv \sin \left(\gamma_{n} y+\right.$ $\left.\gamma_{n}^{\prime}\right)$, where $\tan \gamma_{n}^{\prime}=\gamma_{n} / N$, (0 $\left.\leq \gamma_{n}^{\prime} \leq \pi / 2\right)$, satisfy the second of Eqs. (28) if $\gamma_{n}(n=$ $0,1,2, \cdots)$, is a root of Eq. (29) with $l^{\prime}$ for $l$. Adopting the double suffix notation defined above, $\phi_{m, n}=X_{m}(x) Y_{n}(y)$ satisfies Eq. (21) with the results

$$
\Lambda_{m, n}=\beta_{m}^{2}+\gamma_{n}^{2}
$$

and

$$
\delta_{m, n}(m, n)=\frac{1}{4} l l^{\prime}\left(\frac{\beta_{m}^{2}+N^{2}+2 N / l}{\beta_{m}^{2}+N^{2}}\right)\left(\frac{\gamma_{n}^{2}+N^{2}+2 N / l^{\prime}}{\gamma_{n}^{2}+N^{2}}\right) .
$$

We can now obtain a formula for $b_{p, a}(m, n)$, given by Eq. (27), in the form

$$
b_{p, q}(m, n)=\frac{1}{4}\left\{c_{|m-p|,|n-a|}-c_{|m-p|, n+a}+c_{m+p, n+a}-c_{m+p,|n-a|}\right\}
$$

where

$$
\begin{aligned}
c_{m+p, n+a}=\int_{0}^{l} \int_{0}^{l \prime} r \cos \left\{\left(\beta_{m}\right.\right. & \left.+\beta_{p}\right) x \\
& \left.+\left(\beta_{m}^{\prime}+\beta_{p}^{\prime}\right)\right\} \cos \left\{\left(\gamma_{n}+\gamma_{a}\right) y+\left(\gamma_{n}^{\prime}+\gamma_{a}^{\prime}\right)\right\} d x d y,
\end{aligned}
$$

and a suffix $|m-p|$, say, involves a change of sign between elements with suffixes $m$ and $p$ on the right hand side of Eq. (34). Note that this notation is defined only for 
compound suffixes. A term $c_{m, n}$ has no meaning in the general case, although exceptionally it has in the following limiting cases. Putting $N=\infty$, the constant wall temperature case, we have $\beta_{m}^{\prime} \equiv 0, \gamma_{n}^{\prime} \equiv 0, \beta_{m}=m \pi / l, \gamma_{n}=n \pi / l^{\prime}$ and $\delta_{m, n}(m, n) \Lambda_{m, n}=\left(\pi^{2} / 4 \alpha\right)$ $\left(m^{2}+\alpha^{2} n^{2}\right)$. The expansion (22) is now in the form of a double Fourier sine series in $\left(0 \leq x \leq l, 0 \leq y \leq l^{\prime}\right)$. The coefficients given by Eq. (34) can now be identified with members of the set whose general term is

$$
d_{i, j}=\int_{0}^{l} \int_{0}^{l^{\prime}} r \cos (i \pi x / l) \cos \left(j \pi y / l^{\prime}\right) d x d y, \quad(i, j=0,1,2 \cdots),
$$

that is, they may be associated with the coefficients of the double Fourier cosine expansion of $r(x, y)$ in $\left(0 \leq x \leq l, 0 \leq y \leq l^{\prime}\right)$. Equation (33) still holds identically with $d$ for $c$. On the other hand, if $N=0$ the only difference is that $\beta_{m}^{\prime} \equiv \gamma_{n}^{\prime} \equiv \pi / 2$ and we find that we can write $d$ in place of $c$ in Eq. (33) provided we change the negative signs in this equation. This case is of no interest in the present problem but may be so in other applications since the above formulae are true for arbitrary $r(x, y)$. In the present problem $r(x, y)$ is found from Eq. (3) to be

$$
r(x, y)=\frac{1}{f_{0}} \sum_{i=1}^{\infty} \sum_{j=1}^{\infty} i^{-1} j^{-1}\left(i^{2}+\alpha^{2} j^{2}\right)^{-1} \sin (i \pi x / l) \sin \left(j \pi y / l^{\prime}\right),
$$

where

$$
f_{0}=\left(4 / \pi^{2}\right) \sum_{i=1}^{\infty} \sum_{j=1}^{\infty} i^{-2} j^{-2}\left(i^{2}+\alpha^{2} j^{2}\right)^{-1}
$$

and, because $r(x, y)$ is symmetrical about both $x=l / 2$ and $y=l^{\prime} / 2, i$ and $j$ are restricted to be odd integers only. Substitution into Eq. (34) yields a formula for $c_{m+p, n+q}$ which is expressed as a double summation with respect to $i$ and $j$ but which can be summed with respect to one of these variables of summation to give a rapidly convergent single series. We also find that, because of the symmetry present in $r(x, y), c_{m+p, n+q}$ is zero under certain circumstances. Let us associate odd integers with the roots of the first of Eqs. (30) and even integers with the roots of the second. Then it is readily shown that $c_{m+p, n+q}$ is non-zero only if $m+p$ and $n+q$ are both even integers, and that the same applies to each of the other three coefficients in Eq. (33). The formula for the non-zero coefficients is

$$
c_{m+p, n+q}=c_{m+p, n+q}^{\prime} \sum_{j=1}^{\infty} \frac{\pi \alpha j \tan \left\{\frac{1}{2}\left(\beta_{m}+\beta_{p}\right) l\right\}-\left(\beta_{m}+\beta_{p}\right) l \tanh \left(\frac{1}{2} \alpha j \pi\right)}{j\left\{\alpha^{2} j^{2} \pi^{2}+l^{2}\left(\beta_{m}+\beta_{p}\right)^{2}\right\}\left\{\pi^{2} j^{2}-l^{\prime 2}\left(\gamma_{n}+\gamma_{a}\right)^{2}\right\}},
$$

where $c_{m+p, n+a}^{\prime}=\pi^{3} l^{\prime} \cos \left(\beta_{m}^{\prime}+\beta_{p}^{\prime}\right) \cos \left(\gamma_{n}^{\prime}+\gamma_{q}^{\prime}\right) / \alpha f_{0}\left(\beta_{m}+\beta_{p}\right)$, and $j$ is odd. The other three coefficients required in Eq. (33) are obtained from Eq. (37) by appropriate changes of sign. Since, for all values of $N$, the roots of Eqs. (30) all approach values which are integral multiples of $\pi / l$, it is clear that the quadruple sum of $b_{p, a}(m, n)$ with respect to $p, q, m$, and $n$ converges absolutely so that $\Delta(\lambda)$ converges.

We may now consider the special nature of the eigenfunctions derived from the solutions of the algebraic equations. Since $b_{p, q}(m, n)$, which is the coefficient of $a_{p, q}$ in the $(m, n)$ th equation, is non-zero only if $m+p$ and $n+q$ are both even it follows at once that the equations break up into four independent sets. Since also $a_{p, a}$ is the coefficient of $X_{p}(x) Y_{q}(y)$ in the expansion for $\theta$ there are four corresponding independent sets of eigenfunctions which exhibit the alternative properties $(i)$ symmetry about both 
$x=l / 2$ and $y=l^{\prime} / 2$, (ii) anti-symmetry about $x=l / 2$ and $y=l^{\prime} / 2$, (iii) symmetry about $x=l / 2$ with anti-symmetry about $y=l^{\prime} / 2$ and (iv) the opposite of the last case. Of these only (i) concerns us in this problem since by Eq. (13) only these solutions give non-zero coefficients in Eq. (12). The special case $\alpha=1$ needs further consideration since here, in addition to the matrix symmetry $b_{p, q}(m, n)=b_{m, n}(p, q)$ present in all cases, we also have $b_{p, q}(m, n)=b_{a, p}(n, m)$. It follows that if an ordered set of coefficients $\left\{a_{p, a}\right\}$ with a given eigenvalue $\lambda=\lambda_{n}$ satisfies the algebraic equations then so, with the same eigenvalue, does the set $\left\{a_{a, p}\right\}$ obtained by interchange of $a_{a, p}$ with $a_{p, q}$. That is, if $\Theta_{n}^{\prime}(x, y)=\sum_{p=1}^{\infty} \sum_{a=1}^{\infty} a_{p, q} X_{p}(x) Y_{q}(y)$ is a solution then a linearly independent solution is $\Theta_{n}^{\prime}(y, x)=\sum_{p=1}^{\infty} \sum_{a=1}^{\infty} a_{a, p} X_{p}(x) Y_{q}(y)$ and, since the eigenvalues are equal, these solutions may not satisfy the orthogonality property given by Eq. (10), which would invalidate Eq. (13). On the other hand the sum and difference of these solutions are both themselves solutions and we can write their contribution to the right hand side of Eq. (12) as

$$
Q_{n}\left\{\Theta_{n}^{\prime}(x, y)+\Theta_{n}^{\prime}(y, x)\right\}+Q_{n}^{\prime}\left\{\Theta_{n}^{\prime}(x, y)-\Theta_{n}^{\prime}(y, x)\right\} .
$$

Multiplying Eq. (12) by $w\left\{\Theta_{n}^{\prime}(x, y)-\Theta_{n}^{\prime}(y, x)\right\}$ and integrating over $D^{\prime}$ we find at once that $Q_{n}^{\prime}=0$ and in the remaining term, considered as a single eigenfunction with eigenvalue $\lambda_{n}$, the terms $X_{p}(x) Y_{q}(y), X_{q}(x) Y_{p}(y)$ occur with equal coefficients. It follows that in the case $\alpha=1$ we can ab initio put $a_{p, q}=a_{a, p}(p, q=1,3,5, \cdots)$, in the algebraic equations and that each eigenfunction derived from this reduced set of equations corresponds to a unique term in the expansion (12) with $a_{n}$ given as usual by Eq. (13). We have, of course, assumed that the $\lambda_{n}$ of the reduced set of equations are themselves distinct. It follows also in this case that the expansion (8) consists only of functions for which $\Theta(x, y) \equiv \Theta(y, x)$, which we would expect physically.

It remains only, in the general case, to evaluate $a_{n}$ from each computed $\theta_{n}$. Substituting in Eq. (13) from Eq. (17) we have

$$
Q_{n}=-\frac{1}{A^{\prime} \lambda_{n}} \int_{C^{\prime}} \frac{\partial \Theta_{n}}{\partial \nu^{\prime}} d s^{\prime}
$$

and since

$$
\int_{C^{\prime}} \frac{\partial \Theta_{n}}{\partial \nu^{\prime}} d s^{\prime}=-2\left[\int_{0}^{l}\left\{\frac{\partial \Theta_{n}}{\partial y}\right\}_{y=0} d x+\int_{0}^{l \prime}\left\{\frac{\partial \Theta_{n}}{\partial x}\right\}_{x=0} d y\right]
$$

then

$$
Q_{n}=\frac{16 \alpha N^{2}}{(1+\alpha)^{2} \lambda_{n}} \sum_{p=1}^{\infty} \sum_{a=1}^{\infty} \sin \beta_{p}^{\prime} \sin \gamma_{a}^{\prime}\left(\beta_{p}^{-2}+\gamma_{a}^{-2}\right) a_{p, a}^{(n)} .
$$

A more rapidly convergent formula is found by substituting directly for $\theta_{n}$ into Eq. (13) but it is more complicated except in the special case $N=\infty$, in which it becomes

$$
\widehat{Q}_{n}=\frac{1}{4 f_{0}} \sum_{p=1}^{\infty} \sum_{a=1}^{\infty} p^{-1} q^{-1}\left(p^{2}+\alpha^{2} q^{2}\right)^{-1} a_{p, a}^{(n)} .
$$

In these formulae $\left\{a_{p . a}^{(n)}\right\}$ are the particular set of coefficients which refer to $\theta_{n}$ and which satisfy Eq. (11). In practice, solutions of the algebraic equations have been computed by arbitrarily putting one coefficient equal to unity. If $\left\{A_{p, q}^{(n)}\right\}$ is such a solution and we put $A_{p, q}^{(n)}=\mathfrak{N}_{n} a_{p, a}^{(n)}$, then $\mathscr{N}_{n}$ is found from Eq. (11). From Eq. (9) we 
have $\iint_{D^{\prime}} w \Theta_{n}^{2} d x d y=-\left(w_{0} / \lambda_{n}\right) \iint_{D^{\prime}}, \Theta_{n} \nabla^{2} \Theta_{n} d x d y$ so that

$$
\mathfrak{T}_{n}^{2}=\frac{4 \alpha}{\lambda_{n}(1+\alpha)^{2}} \sum_{p=1}^{\infty} \sum_{a=1}^{\infty} \delta_{p, q}(p, q)\left(\beta_{p}^{2}+\gamma_{q}^{2}\right)\left[A_{p, a}^{(n)}\right]^{2} .
$$

Computational results. For a given value of $\alpha$ the computation from the algebraic equations of the first few eigensolutions, arranged in ascending order of $\lambda$, is a standard problem. We have used relaxation methods in conjunction with Rayleigh's principle, $\lambda_{n}$ for a given eigensolution being estimated by the Rayleigh quotient

$$
\begin{aligned}
\lambda_{n} & =-\int_{0}^{l} \int_{0}^{l^{\prime}} \Theta_{n} \nabla_{1}^{2} \Theta_{n} d x d y / \int_{0}^{l} \int_{0}^{l^{\prime}} r \Theta_{n}^{2} d x d y, \\
& =\sum_{p=1}^{\infty} \sum_{a=1}^{\infty} \delta_{p, q}(p, q)\left(\beta_{p}^{2}+\gamma_{a}^{2}\right)\left[A_{p, q}^{(n)}\right]^{2} / \sum_{i=1}^{\infty} \sum_{j=1}^{\infty} \sum_{p=1}^{\infty} \sum_{q=1}^{\infty} b_{p, q}(i, j) A_{i, i}^{(n)} A_{p, a}^{(n)} .
\end{aligned}
$$

Since the diagonal elements strongly dominate the matrix, good initial approximations to the eigenvalues are found by equating each successive diagonal to zero giving estimates $\lambda_{m, n}=\delta_{m, n}(m, n)\left(\beta_{m}^{2}+\gamma_{n}^{2}\right) / b_{m, n}(m, n)$ where, for obvious reasons, we temporarily adopt double-suffix notation for the $\lambda$ 's. We obtain an initial estimate of the $(m, n)$ th eigenfunction using this approximation for $\lambda_{m, n}$, arbitrarily putting $A_{m, n}^{(m, n)}=1$ and using the other equations except the $(m, n)$ th to estimate the remaining coefficients in the form

$$
A_{p, q}^{(m, n)} \bumpeq b_{m, n}(p, q) /\left[\frac{\delta_{p, q}(p, q)\left(\beta_{p}^{2}+\gamma_{q}^{2}\right)}{\delta_{m, n}(m, n)\left(\beta_{m}^{2}+\gamma_{n}^{2}\right)} b_{m, n}(m, n)-b_{p, q}(p, q)\right] .
$$

This approximation is computed until the coefficients attain some prescribed order of smallness acceptable as error. It is then improved by the relaxation process until a final accepted solution is reached upon which we impose the criteria that no coefficient larger than the agreed order of smallness be omitted, nor must any omitted coefficient affect, to the agreed working accuracy, the value of any included coefficient. The eigenfunctions themselves are of little physical interest but to illustrate the numerical analysis we give in Table 1, correct to four decimal places, the first two in the case $\alpha=1, N=\infty$. The physical results of interest depend on the expansions (14), (18) and (19). The $\leftrightarrow$ 's converge fairly rapidly and we need only the first few terms to describe the physical domain, that is, $z>z_{0}$ (say). It is pointless to extend the solution too near to $z=0$ for since $z=\zeta / d$ Pé

TABLE 1

$\alpha=1, N=\infty$

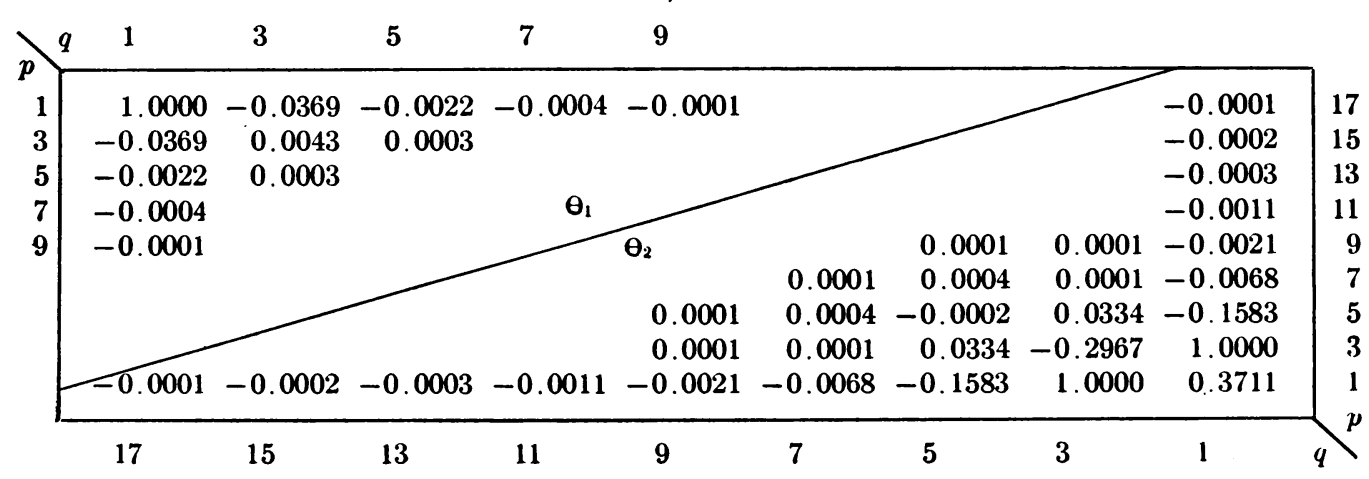


then, for fixed $\zeta$ and a given fluid, this would be equivalent to increasing the Reynolds number beyond the laminar flow range. In our results we have treated the constant. wall temperature case in considerable detail since this is the case dealt with by Clark and Kays (loc. cit.). In Table 2 the first three $\lambda_{n}$ and associated $\Theta_{n}$ are given in each of the cases $\alpha=1,2 / 3,1 / 2,1 / 4$, and 1/8. For the more general radiation boundary condition we have considered only the square duct. The first three terms for $\theta_{M}$ in the case $N=2$ are

$$
\theta_{M}=0.972 \exp (-4.81 z)+0.023 \exp (-47.6 z)+0.003 \exp (-127 z) \cdot+\cdots
$$

while the first only for the cases $N=10,20$ are respectively

$$
\theta_{M}=0.893 \exp (-9.18 z)+\cdots \text { and } \theta_{M}=0.860 \exp (-10.37 z)+\cdots
$$

The eigenvalues are well separated for the square duct so that in fact even the first term describes well the physical domain. Beyond $N=20, \lambda_{1}$ [and hence the important quantity $N u(\infty)$ ] can be calculated to good accuracy from the formula

$$
\frac{\left(\lambda_{1}\right)_{N}}{\left(\lambda_{1}\right)_{N=\infty}} \bumpeq \frac{\beta_{1}^{2}}{\pi^{2}}\left(\frac{\beta_{1}^{2}+N^{2}+2 N}{\beta_{1}^{2}+N^{2}}\right)^{2} \frac{\left[b_{1,1}(1,1)\right]_{N=\infty}}{\left[b_{1,1}(1,1)\right]_{N}} .
$$

This formula is based on the assumption that the correct value of $\lambda_{1}$ for a given $N$ bears the same ratio to $\lambda_{1}$ in the case $N=\infty$ as do the corresponding diagonal estimates of these eigenvalues in the initial approximation given previously since, by Rayleigh's principle, these latter are always over-estimates. For example when $N=20$, Eq. (44) gives $\lambda_{1}=10.42$ against the correct value $\lambda_{1}=10.37$.

Comparison of thermal results. The theory used by Clark and Kays is based on the assumption, previously used by Seban and Shimazaki [7] for turbulent flow in cylinders, that far enough from the thermal inlet

$$
\frac{\partial}{\partial \zeta}\left(\frac{T-T_{1}}{T_{M}-T_{1}}\right)=0
$$

and this is borne out in our work since, for large enough $z, \theta / \theta_{M} \sim \theta_{1}(x, y) / a_{1}$, and is: independent of $z$. Calculated results for the limiting Nusselt number in the cases $\alpha=1$ and 0.5 are given respectively as $N u^{\prime}(\infty)=2.89$ and 3.39 and these compare well with our values of 2.98 and 3.39. No theoretical information on the variation of Nusselt. number in the thermal inlet length is given, but experimental data have been obtained by the authors for the aspect ratios $\alpha=1$ and 0.382 . These largely confirm the theoretical.

TABIE 2

$$
N=\infty
$$

\begin{tabular}{|llllll|}
\hline & & & & & \\
$\alpha$ & 1.000 & 0.667 & 0.500 & 0.250 & 0.125 \\
$\lambda_{1}$ & 11.91 & 12.49 & 13.57 & 17.76 & 22.38 \\
$\lambda_{2}$ & 71.07 & 51.58 & 41.17 & 28.17 & 25.61 \\
$\lambda_{3}$ & 157.9 & 99.71 & 94.93 & 47.82 & 31.81 \\
$\AA_{1}$ & 0.804 & 0.802 & 0.789 & 0.756 & 0.737 \\
$\AA_{2}$ & 0.104 & 0.064 & 0.071 & 0.107 & 0.091 \\
$\AA_{3}$ & 0.014 & 0.043 & 0.020 & 0.028 & 0.034 \\
& & & & & \\
\hline
\end{tabular}




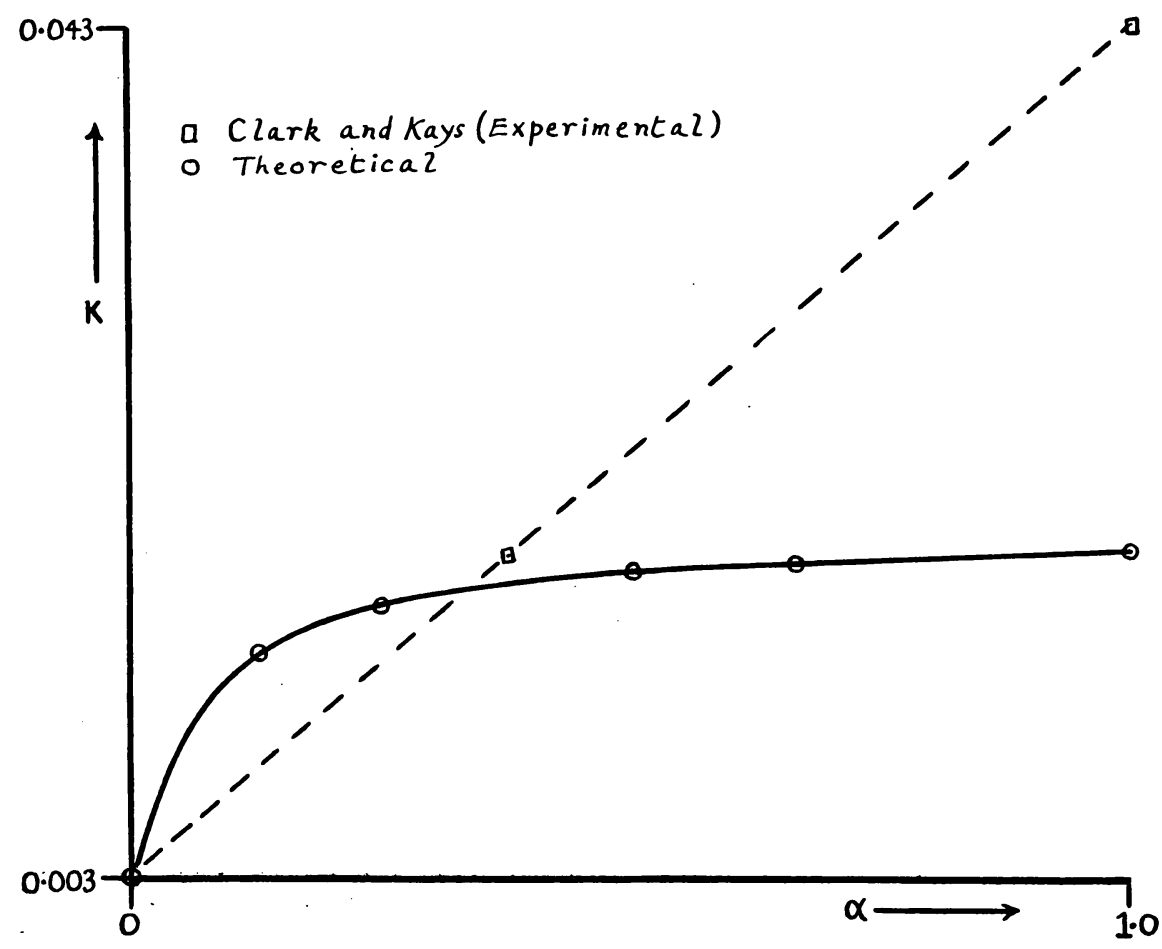

Fig. 1. $K$ against aspect ratio for the case of constant wall temperature.

values of $N u(\infty)$ and also it is found that for small values of $d \mathrm{Pe} / \zeta$ the variation of the logarithmic mean Nusselt number is linear according to the formula

$$
\frac{N u^{\prime}(z)}{N u^{\prime}(\infty)}=1+K\left(\frac{d \mathrm{Pe}^{\prime}}{\zeta}\right)
$$

This linear law follows theoretically from Eq. (20) which gives the theoretical value $K=\left(1 / \lambda_{1}\right) \ln \left(1 / \AA_{1}\right)$. In the case $\alpha=0.382$ the theoretical value $K=0.016$ agrees well with the experimental value 0.017 , but this is not so for $\alpha=1$ where we find $K=0.018$ against the experimental value 0.042 . The theoretical curve for $K$ against $\alpha$ is compared with Clark and Kays tentative linear correlation in Fig. 1. The disagreement is serious, but we must point out that the experimental curve is determined by only two observed points, the result for $\alpha=0$ being theoretical, so that an error in an observed point could give a very different curve. On account of the discrepancy for the square duct we have investigated the radiation boundary condition fully in this case and the results for $K$ against $N^{-1}$ are given in Fig. 2. Clearly the values of $K$ are always lower than those in the constant wall temperature case, so no possible explanation is forthcoming from these results. On the other hand, comparison of experimental and theoretical values of $K$ for the circular cross-section [8] suggests that experimental values may be considerably higher. This may lessen the discrepancy in the square case but, for consistency, the experimental value for $\alpha=0.382$ should also be higher. This is possible since Clark and Kays state that in this case the ratio of duct-length to mean hydraulic depth used in their apparatus could possibly be higher than the assumed value by as much as $100 \%$; 


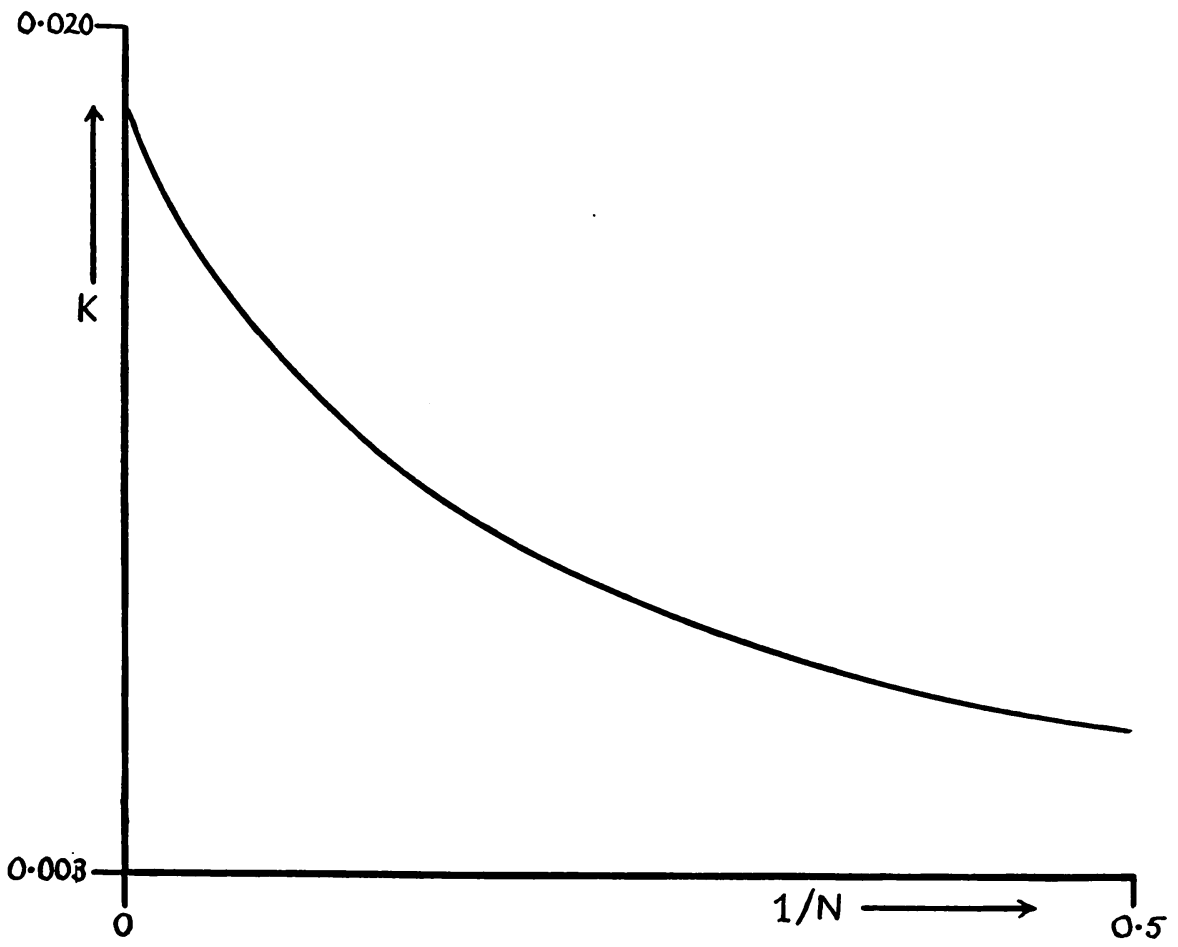

Fig. 2. $K$ against $N^{-1}$ for the square duct.

this would lead to a larger value of $K$. In the absence of more detailed experimental results, however, it is impossible to state precisely the cause of the disagreement. Finally, we should notice that there is some doubt regarding the theoretical value for $K$ near the limiting case $\alpha=0$. We consider only $N=\infty$ but the general case is similar. If we keep the side of the duct parallel to the $\xi$-axis fixed and let the other become large then $\partial^{2} \theta / \partial y^{2} \rightarrow 0, w / w_{0} \rightarrow 24 x(1 / 2-x)$ in Eq. (4). The solution for $\theta$ may now be written $\theta(x, z)=\sum_{m=1}^{\infty} Q_{m}^{\prime} \vartheta_{m}(x) \exp \left(-\lambda_{m}^{\prime} z\right)$ where $\vartheta^{\prime \prime}+24 \lambda^{\prime} x(1 / 2-x) \vartheta=0$ with $\vartheta(0)=$ $\vartheta(1 / 2)=0$. Now each $\vartheta_{m}(x)$ can be written as

$$
\frac{4}{\pi} \sum_{n=1}^{\infty} \vartheta_{m} \frac{\sin \left(n \pi y / 1^{\prime}\right)}{n}, \quad(n=1,3,5 \cdots)
$$

that is, it can be considered as a sum of functions $\theta_{m, n}(x, y)$ with identical eigenvalues $\lambda_{m}^{\prime}$ and these latter functions can, for varying $m$ and $n$, be identified with the limiting solutions, here written in double-suffix notation, of our previous algebraic equations. It is therefore clear that when $\alpha=0$ the value of $B_{1}$ to be used in Eq. (20) should be the sum $\sum_{n=1}^{\infty} \Theta_{1, n},(n=1,3,5, \cdots)$, of the $\Re^{\prime}$ 's associated with $\Theta_{1, n}(x, y)$ and since it is easily shown that $\Theta_{1, n}=n^{-2} \Theta_{1,1}$ this sum is $\pi^{2} \Theta_{1,1} / 8$. When $\alpha \neq 0$ and the $\lambda$ 's are all distinct we should use $B_{1,1}$ which, in double-suffix notation, corresponds to the smallest $\lambda$. There is therefore some doubt as to the correct procedure near $\alpha=0$ but, in practice, it can make very little difference to Fig. 1 since $K$ is so small at this end of the curve. 
Acknowledgment. We acknowledge a grant in aid of this work by the Royal Society. A preliminary (unpublished) account was given by one of us (A.Mc.D.M.) to the IXth International Congress of Mechanics and Applied Mathematics, Brussels, 1956.

\section{REFERENCES}

1. S. H. Clark and W. M. Kays, Trans. A.S.M.E. 75, 859-866 (1953)

2. W. M. Hampton, Nature 157, 481 (1946)

3. R. Courant and D. Hilbert, Methods of mathematical physics, vol. 1, Interscience publishers, New York, 1953

4 M. Jakob, Heat transfer, vol. 1, John Wiley, New York, 1949

5. W. E. Milne, Numerical solution of differential equations, John Wiley, New York, 1953, p. 114

6. E. T. Whittaker and G. N. Watson, Modern analysis, Cambridge, 1927, pp. 36, 37, 417

7. R. A. Seban and T. T. Shimazaki, Trans. A.S.M.E. 73, 803-809 (1951)

8. W. M. Kays and A. L. London, Trans. A. S. M. E. 74, 1179-1189 (1952) 